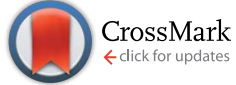

Cite this: RSC Adv., 2015, 5, 39760
Received 19th March 2015

Accepted 23rd April 2015

DOI: $10.1039 / c 5 r a 04880 j$

www.rsc.org/advances

\section{MR imaging and targeting of human breast cancer cells with folate decorated nanoparticles $\uparrow$}

\author{
Paolo Arosio, ${ }^{\text {*a }}$ Francesco Orsini, ${ }^{a}$ Anna M. Piras, ${ }^{b}$ Stefania Sandreschi, ${ }^{b}$ \\ Federica Chiellini, ${ }^{\mathrm{b}}$ Maurizio Corti, ${ }^{\mathrm{c}}$ Marc Masa, ${ }^{\mathrm{d}}$ Marta Múčková, ${ }^{\mathrm{e}}$ \\ Ľudmila Schmidtová, ${ }^{e}$ Costanza Ravagli, ${ }^{f}$ Giovanni Baldi, ${ }^{f}$ Elena Nicolato, ${ }^{g}$ \\ Giamaica Conti, 9 Pasquina Marzola ${ }^{\text {h }}$ and Alessandro Lascialfari ${ }^{\mathrm{a}}$
}

\begin{abstract}
The way of viewing cancer has advanced considerably in the last few decades because of recent progress on two different topics: the knowledge of the mechanisms and characteristics of cancer and the innovation in imaging agent design. In particular the unique properties of cancer that allow differentiation from normal tissue could be employed in multi-functional nanoparticle imaging development. Genetic alterations, either endogenous or induced through gene therapy, are one class of such characteristics. At the same time proteomic differences such as overexpressed surface receptors are another targetable feature, used for enhanced nanoparticle retention. The here proposed magnetic nanoparticle (with biocompatible coating) was designed to target the human breast MDA-MB-231 tumor induced on a nude mice model. With the aim of developing a theranostic agent, the overexpression of folate protein receptor in breast cancer cells was exploited, decorating with folate an organic nanocarrier loaded with magnetite nanoparticles that acts as a diagnostic MRI (Magnetic Resonance Imaging) contrast agent, and Paclitaxel (PTX) as antitumoral drug. A high uptake of nanoparticles and remarkable effect on in vivo MRI images show the targeting ability of our compound and its prolonged retention in tumor tissues. Due to the presence of PTX, the developed nanocarrier may potentially be used also for therapeutic purposes.
\end{abstract}

\section{Introduction}

Any strategy that improves tumour penetration can potentially result in enhanced clinical efficacy of nanomedicines over existing therapeutics. Multifunctional nanoparticles have been developed for disease imaging via passive targeting, but recent advances in nanotechnology have enabled cellular-specific targeting, drug delivery and multi-modal imaging using these nanoplatforms. The aim of this interdisciplinary research is to

${ }^{a}$ Department of Physics, Università degli Studi di Milano and Consorzio INSTM, Milano Unit, I-20133 Milano, Italy. E-mail: paolo.arosio@guest.unimi.it

${ }^{b}$ Laboratory of Bioactive Polymeric Materials for Biomedical and Environmental Applications (BIOlab), UdR INSTM, Department of Chemistry \& Industrial Chemistry, University of Pisa, Pisa, Italy

${ }^{c}$ Department of Physics, Università degli Studi di Pavia and Consorzio INSTM, Pavia Unit, Italy

${ }^{d}$ Leitat, Centro Tecnológico C/de la Innovació, 2, 08225 Terrassa, Barcelona, Spain ${ }^{e}$ Hameln rds a.s., Horná 36, 90001 Modra, Slovak Republic

${ }^{f}$ CERICOL Colorobbia, via Pietramarina 123, 50053 Sovigliana-Vinci, Firenze, Italy ${ }^{g}$ Department of Neurological and Movement Sciences, University of Verona, Verona, Italy

${ }^{h}$ Department of Computer Science, University of Verona, Verona, Italy

$\dagger$ Electronic supplementary information (ESI) available: The details of the nanoparticle characterization not specified in Materials and Methods (AFM image of Block-MNP-FA and NMRD relaxivity curves of NPs) are reported in ESI. See DOI: $10.1039 / \mathrm{c} 5 \mathrm{ra} 04880 \mathrm{j}$ develop targeted multifunctional nanoparticles, serving as both imaging agents and drug carriers that can effectively pass biological barriers, for diagnosis, staging and treatment of tumours. $^{1-6}$

To achieve cancer-targeted drug delivery and in situ treatments, several methods have been attempted among which one strategy is to pursue the targeting of unique molecular markers that are specifically overexpressed within the cancerous tissues. Among the agents directed to membrane-bound tumour associated receptors, folic acid (FA) has been widely utilized as a ligand for the selective targeting and delivery of macromolecular drugs into tumour cells. In fact it is well known that folate receptor (FR), the folate-binding protein, is overexpressed on the surface of many human epithelial cancer cells and is rarely found on normal cell surfaces..$^{7-9} \mathrm{FA}$ is appealing because it is useful for targeting cell membranes and enhancing endocytosis of nanoparticles via the FRs. Recycling of FRs in target cells can lead to the transport of more FA conjugates. ${ }^{10}$ So far, many researchers have reported on FA-conjugated polymeric micelles, macromolecules, nanoparticles, and liposomes for the targeted delivery of anticancer agents or genes. ${ }^{11-14}$ Among the different types of nanoparticles in nanomedicine, the increasing research interest on magnetic nanoparticles (MNPs) is justified by their unique magnetic and chemical properties combined with a substantial biocompatibility. ${ }^{15-19}$ Besides their employment in 
Magnetic Resonance Imaging (MRI) ${ }^{20-23}$ MNPs are interestingly involved in therapy as hyperthermia agents. ${ }^{24-27}$ In particular several iron oxide nanoparticles were approved and used for the clinical practice as negative MRI Contrast Agents, (i.e. Endorem ${ }^{\circledR}$ and Resovist $\left.{ }^{\circledR}\right)$ and as magneto-hyperthermic Agents (NanoTherm ${ }^{\mathrm{TM}}$ ).

Here we report on further steps in this direction. The investigated multifunctional MNPs are constituted by a core of magnetite $\left(\mathrm{Fe}_{3} \mathrm{O}_{4}\right)$ coated with random multiblock poly(esterether-urethane)s copolymers (PEEU) of PCL and PEG. The synthetic polymers were obtained under scaled-up conditions ${ }^{28}$ and the nanoparticle formulations were assessed in view of easy high quality upscaling for potential clinical applications. Our attention was mainly focused on nanoparticles (NPs) decorated with folate and loaded with paclitaxel (PTX) as antitumoral drug while the $\mathrm{Fe}_{3} \mathrm{O}_{4}$ inorganic magnetic core allows the system to act as diagnostic MRI contrast agent. PTX, one of the most successful anticancer drugs, is the first of a new class of microtubule-stabilizing agents, and has demonstrated significant antitumor activity in clinical trials against a broad range of solid tumours, especially against non-small-cell lung cancer, metastatic breast cancer, refractory ovarian cancer, and hepatic cancer. $^{29,30}$

On the other hand FRs exist in three major forms, namely FR- $\alpha$, FR- $\beta$, and FR- $\gamma$. In particular we focused on the FR- $\alpha$ form because it is further qualified as a tumour-specific target, overexpressed by many types of tumour cell, including those of the ovary, brain, kidney, breast, myeloid cells and lung.

The developed NPs decorated (or not) with folate and loaded (or not) with PTX have been characterized in vitro (cytotoxicity evaluation) and in vivo (biodistribution and efficacy in tumor growth inhibition). Folate decorated NPs loaded with magnetite demonstrated full cytocompatibility and no toxicity. In vivo experiments on nude mice, where human FR- $\alpha(+)$ MDA-MB-231 tumor was implanted, showed the presence of nanoparticles uptake by means of a remarkable effect on $T_{2}^{*}$ weighted MRI images also at 24 hours after injection, meaning that the nanoparticles decorated with folate show targeting ability and a prolonged retention in FR- $\alpha(+)$ tumor tissues. Remarkably the presence of PTX on the nanocarrier targeting the breast tumor opens the way to localized therapy.

\section{Results and discussion}

\section{Preparation and characterization of nanoparticles}

The nanocarriers (from now on called NPs) were prepared according to a common procedure based on nanoprecipitation/ solvent displacement techniques. In details, the PEGCL copolymer $^{28}$ was dissolved in acetone/DMSO 92.5/7.5 at a concentration of $1 \% \mathrm{w} / \mathrm{v}$ and added dropwise to deionised water (acetone/water are 0.4 by volume). The resultant milky suspension was stirred under hood for four hours at $40{ }^{\circ} \mathrm{C}$ for solvent removal. The polymeric solution containing folate was prepared by using a PEGCL/PEGCL-FA (87/13 wt) copolymers blend. The PEGCL-FA copolymer (see its structure in Chart 1) was synthesized under mild conditions, in absence of catalyst, and in presence of a small excess of coupling agent useful for the bonding of the terminal functional block. The folate functional blocks were simply prepared by DCC coupling reaction with a yield of $65 \%$. The UV spectroscopy analysis indicated one spacer per FA unit (76.4 $\pm 1.7 \mathrm{wt} \%$ of FA), confirming that the coupling took place only for one of the two carboxyl groups of the FA. The main characteristics of the synthesized PEGCL-FA copolymer were Mn $15240 \mathrm{~g} \mathrm{~mol}^{-1}$ (PI 1.8), PCL/PEG ratio 2.5, FA content of $10.4 \pm 0.7 \mathrm{wt} \%$.

To obtain the final product, a DEG suspension of $\mathrm{Fe}_{3} \mathrm{O}_{4}$ nanoparticles and an ethanol solution of PTX $\left(10 \mathrm{mg} \mathrm{ml}^{-1}\right)$ were added to the polymeric solution before dropping. The applied polymer/MNP and polymer/PTX ratio were $18 / 1$ and $40 / 1$ by weight, respectively. The NPs were purified by centrifugation at $10000 \mathrm{~g}$ for $40 \mathrm{~min}$, at $4{ }^{\circ} \mathrm{C}$, and re-dispersed in water. All NP formulations were performed at least in triplicates, in batches of $10 \mathrm{ml}$ containing at least $10 \mathrm{mg} \mathrm{ml}^{-1}$ of NPs each.

The nanoprecipitation/solvent displacement protocol applied for the preparation of NPs led to homogeneous water suspensions containing nanosized particles with monomodal diameter distribution and average diameter in the range of 100$140 \mathrm{~nm}$, with a fairly narrow standard deviation (15-40 nm) (Table 1).

The procedure applied for the preparation of the folate decorated NPs lead to highly reproducible batches in terms of diameter distribution and loading content of MNP and PTX. The NPs were produced in batches of $10 \mathrm{ml}$ containing at least $10 \mathrm{mg} \mathrm{ml}^{-1}$ of NPs each. AFM images confirmed the spherical shape of the NPs as well as the diameter size range calculated from Light Scattering (LS) analysis (Fig. 1 and ESI $1 \dagger$ ).

The zeta potential measurements were used to determine the surface features of the NPs, particularly with respect to the loading of PTX and magnetite, and the exposure of the carboxyl groups belonging to the folate-terminal. The surfaces of the NPs are negatively charged, due to the chain of the copolymer bearing no dissociating groups and having a partially hydrophobic character which contributes to a preferred absorption of the chloride anions in solution. The use of folate-functionalised copolymer significantly changed the zeta potential modulus of the resultant NPs by increasing the modulus of the charge, also for magnetite and PTX loaded particles. The NPs relevant to the adopted copolymers blend (87/13 of PEGCL/PEGCL-FA ratio) are known to display 200 functional folate $\gamma$-conjugates on their surface, determining a selective targeting to FR- $\alpha(+)$ tumor cell lines. ${ }^{14}$

The loading of PTX induced a considerable reduction of the modulus of the Zeta potential, attributed to the adsorption of the hydrophobic drug also onto the surface of the carrier. On the contrary, the loading of MNPs caused an increase of the modulus, leading to more negatively charged surfaces. Actually, the isoelectric point of MNP is generally around pH 6.5 (ref. 32) meaning that at the $\mathrm{pH}$ of analysis $(\mathrm{pH} 7.4)$, the magnetite was negatively charged. The complete nanosystem, involving the loading of MNPs and PTX and the targeting moieties to FRs (Block-P-MPN-FA) had a Zeta potential of $-14.8 \mathrm{mV}$, in agreement with the observed trends. Additionally, the Loading Efficiency (LE\%) of PTX in presence of PEGCL-FA was slightly higher than the corresponding not functionalised NPs, mainly 


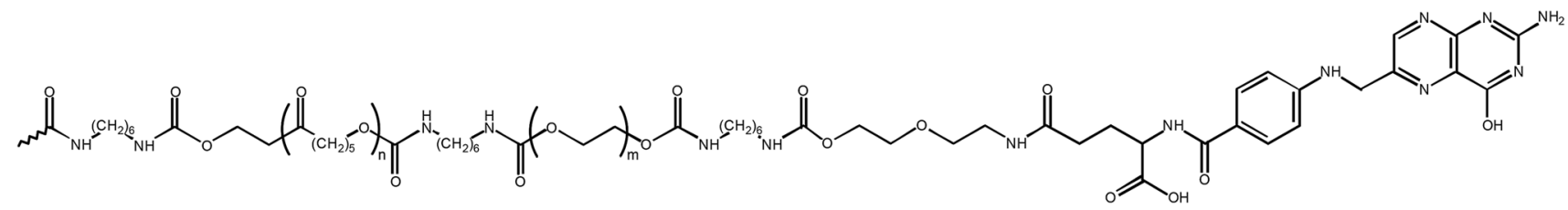

Chart 1 PEGCL-FA copolymer structure.

Table 1 Dimension, zeta-potential and \% weight of PTX and $\mathrm{Fe}_{3} \mathrm{O}_{4}$ of the prepared NPs

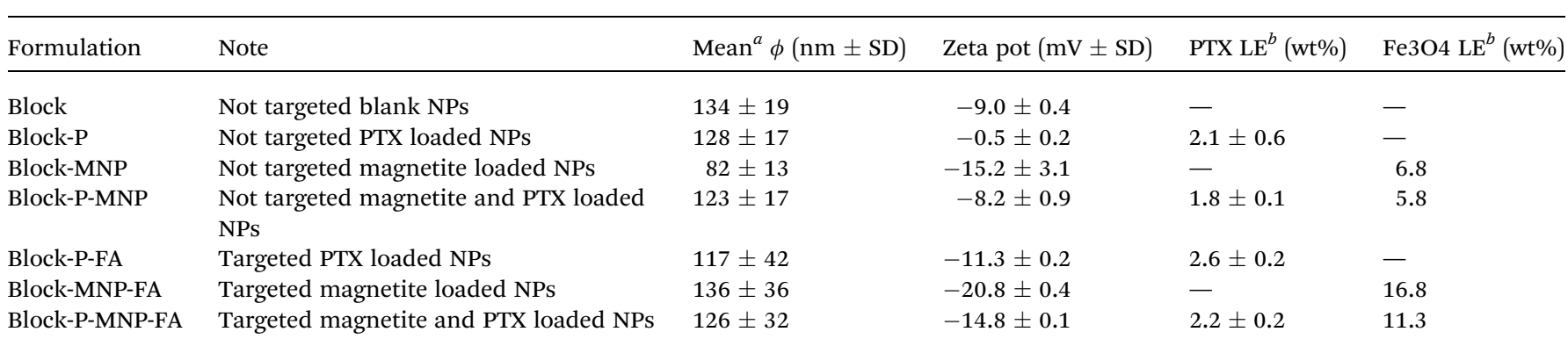

${ }^{a}$ The polydispersity index of the diameter distribution peaks was $0.02 .{ }^{b} \mathrm{LE}=$ Loading Efficiency.
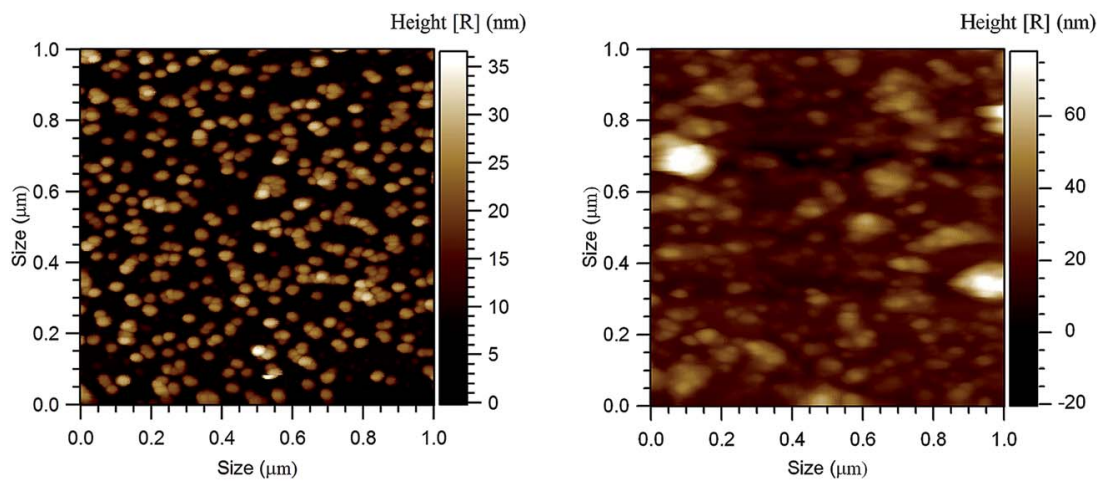

Fig. 1 Topography AFM images of magnetite loaded (Block-MNP, left) and magnetite and PTX loaded targeted NPs (Block-P-MNP-FA, right).

due to a reduced adsorption of the hydrophobic drug on the more hydrophilic surface, which also promoted its inclusion into the PCL hydrophobic domains. Concerning the loading of magnetite NPs, it is known that carboxyl moieties have a good affinity for MNPs and several molecules containing carboxyl groups or derivatives are commonly adopted as stabilizers of MNP. ${ }^{34}$ As result, the LE\% of MNPs was definitely increasing when the PEGCL-FA polymer was included in the formulation of the carrier (Table 1).

The NMR relaxometric characterization of the NPs was carried out on the samples Block-MNP, Block-MNP-FA and Block-P-MNP-FA and compared to the commercial contrast agent Endorem ${ }^{\circledR}$. The analysis of $r_{1}$ and $r_{2}$ at the most used clinical fields i.e. $\sim 0.2 \mathrm{~T}(9.5 \mathrm{MHz}), 0.5 \mathrm{~T}(\sim 21 \mathrm{MHz})$ and $\sim 1.5 \mathrm{~T}$ $(\sim 60 \mathrm{MHz})$ is reported in Table 2 . The whole NMRD longitudinal $\left(r_{1} v s . \nu\right)$ and transverse $\left(r_{2} v s . \nu\right)$ relaxivity curves obtained for all the studied samples are included in ESI (Fig. SI2 $\dagger$ ).

Interestingly, MNPs show $r_{2}$ values higher (surprisingly till 8 times more) than Endorem ${ }^{\circledR}$ in the whole measured frequency range. Being the transverse relaxivity $r_{2}$ the fundamental parameter to test the MRI efficiency for a superparamagnetic material, the obtained data demonstrate that the developed surface decorated PEEU MNPs displayed very good potential for MRI diagnosis. Moreover, it is worth noting that the sample containing the PTX and the targeted moieties (Block-P-MNP-FA) has relaxivity values similar to the ones of the sample without drug (Block-MNP-FA), thus witnessing that no major change of nuclear relaxation occurs when the chemotherapeutic agent is included in the carrier.

\section{Nanoparticles cytocompatibility}

To be considered suitable for biomedical applications, the prepared NPs were submitted to a preliminary in vitro test to assess their biocompatibility. The cytocompatibility was performed on the NPs bearing folate moieties and loaded with magnetite. The PTX loaded systems were omitted due to the intrinsic cytotoxicity of the drug, which is also enhanced by the NPs as reported in previous studies. ${ }^{28}$ Quantitative evaluations 
Table 2 Longitudinal $\left(r_{1}\right)$ and Transverse $\left(r_{2}\right)$ relaxivities $\left(\mathrm{L} \mathrm{mmol}{ }^{-1}\right.$ $\mathrm{sec}^{-1}$ ) of Block-MNP, Block-MNP-FA and Block-P-MNP-FA samples compared to Endorem $®$

\begin{tabular}{lccc}
\hline Sample & @9.5 MHz & @21 $\mathrm{MHz}$ & @60 $\mathrm{MHz}$ \\
\hline $\boldsymbol{r}_{\mathbf{1}}$ & & & \\
Block-MNP & 67.1 & 27.7 & 7.4 \\
Block-MNP-FA & 86.2 & 39.8 & 10.8 \\
Block-P-MNP-FA & 88.6 & 43.3 & 11.8 \\
Endorem® & 26.6 & 21.9 & 9.1 \\
& & & \\
$\boldsymbol{r}_{2}$ & & & 243.7 \\
Block-P-FA & 365.3 & 285.4 & 570.4 \\
Block-MNP-FA & 719.8 & 641.7 & 645.2 \\
Block-P-MNP-FA & 793.2 & 677.5 & 98.6 \\
Endorem ${ }^{\circledR}$ & 102.5 & 92.9 & \\
\end{tabular}

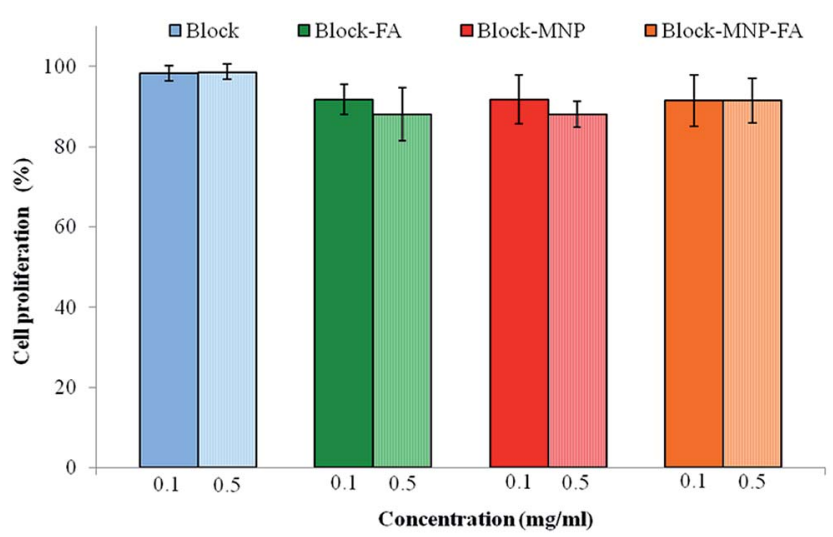

Fig. 2 WST-1 cytocompatibility test performed on plain and magnetite loaded NPs as a function of NPs concentration.

of cell viability, performed by WST-1 assay on balb/3T3 Clone A31 mouse embryo fibroblasts cell line, indicated no toxicity and full cytocompatibility for the developed MNPs loaded NPS (Block-MNP and Block-MNP-FA), in line with results obtained with the plain Block NPs. The cell viability in presence of NPs was comparable to the control cell viability profile up to a NPs concentration of $0.5 \mathrm{mg} \mathrm{ml}^{-1}$, as shown in Fig. 2 .

Despite its synthetic origin and the loading of MNP, the PEEU coating exerted a protective effect which is reflected in the in vitro cytocompatibility.

\section{In vivo biodistribution evaluation}

Biodistribution of the Block-P-MNP-FA was assessed by quantifying the PTX content in various body organs after intravenous (i.v.) administration. The biodistribution study was designed under fasting conditions to eliminate the presence of external FA from the diet. In the excised organs the PTX varied in concentrations according to the experimental time after the injection, but the general distribution of the drug in vital organs and breast cancer tissue was: liver $>$ kidney $>$ spleen $\approx$ lung $>$ tumor, poor in heart and none in brain. The affinity of PTX to the examined tissues is illustrated in Fig. 3 and the basic pharmacokinetic parameters are summarized in Table 3.
The PTX absorption evaluated after the i.v. administration of Block-P-MNP-FA (1 mg kg ${ }^{-1}$ body weight) was low and the maximum plasma concentration $\left(C_{\mathrm{max}}\right)$ was $87.9 \mathrm{ng} \mathrm{ml}^{-1}$.

The clearance of circulating Block-P-MNP-FA from the blood stream was coupled with early uptake by the organs. All examined tissues had a peak at $1 \mathrm{~h}$. At that time, the majority of PTX was extracted from the organs of reticuloendotelial system and from that participating in the elimination of PTX from the body.

A small content of PTX was observed in tumor tissues with $C_{\max }=167 \mathrm{ng} \mathrm{g}^{-1}$ and $\mathrm{AUC}_{0-\infty}=912.85 \mathrm{ng} \mathrm{h}^{-1}$ and about $11 \%$ of $\mathrm{AUC}_{\text {extra; }}$ on the other hand, PTX was detectable in tumor tissues up to 16 hours post dosing. Thus a significant increase in tumor residence time and a subsequent prolongation of half-life elimination corresponding to about 1.5 times that of liver and up to 5 times that of the other vital organs is demonstrated. These results gave evidence of the effect of having the folate moieties exposed on the NPs surface, enhancing the interaction of PTX with tumor cells, and aimed to improve its biodistribution. The data of biodistribution of free PTX in healthy mice (not reported) show a higher plasma $C_{\max }$ for free PTX with similar exposures $\left(\mathrm{AUC}_{0-t}\right.$ and $\left.\mathrm{AUC}_{0-\infty}\right)$. At the same time the clear contrast observed on kinetic parameters between liver and other vital organs (mainly kidney) witness a lower spreading of nanoparticles in the body with respect to the free drug.

In contrast to the results recorded from the administration to healthy mice (5) (ICR mice), the NPs (Block-P-FA) administered in tumor bearing mice displayed a reduced uptake in vital organs, leading to lower content over extended period (AUCs). In tumor bearing mice, the plasma levels of PTX decreased faster than in ICR mice, and the exposures (AUCs) were slightly lower. When tissue distribution was examined $30 \mathrm{~min}$ after i.v. administration, PTX was detected in most of the tissues assessed, mainly in liver, which drove its rapid clearance from blood and facilitated the distribution to the organs of RES and organs of elimination.

\section{Preclinical study of tumor growth inhibition by drug loaded NPs}

The efficacy of Block-P-MNP-FA in metastasis inhibition was compared to that of PTX alone when administered by

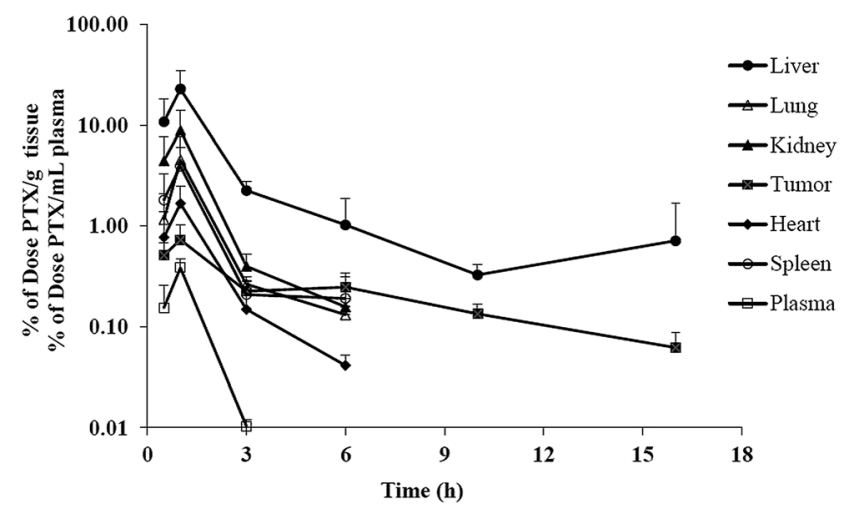

Fig. 3 Affinity of PTX to the plasma, the vital organs and tumor tissue after i.v. administration of Block-P-MNP-FA in tumor breeding mice with breast cancer. 
Table 3 Selected pharmacokinetic parameters in plasma, vital organs and tumor of MDA-MB-231 tumor bearing mice ${ }^{a}$

\begin{tabular}{|c|c|c|c|c|c|c|c|c|c|}
\hline Tissue & $\begin{array}{l}C_{\max } \\
\left(\mathrm{ng} \mathrm{g}^{-1}\right)\end{array}$ & $T_{\max }(\mathrm{h})$ & $\begin{array}{l}\mathrm{AUC}_{0-t} \\
\left(\mathrm{ng} \mathrm{h} \mathrm{g}^{-1}\right)\end{array}$ & 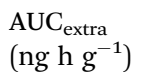 & $\begin{array}{l}\mathrm{AUC}_{0-\infty} \\
\left(\mathrm{ng} \mathrm{h} \mathrm{g}^{-1}\right)\end{array}$ & $\% A U C_{\text {extra }}(\%)$ & $L_{\mathrm{z}}\left(\mathrm{h}^{-1}\right)$ & $T_{1 / 2}(\mathrm{~h})$ & MRT (h) \\
\hline Plasma & $* 87.9$ & 1 & ${ }^{\#} 88.4$ & - & - & - & - & - & - \\
\hline Kidney & 2030 & 1 & 2505.9 & 56.99 & 2562.9 & 2.2 & 0.74 & 0.94 & 1.57 \\
\hline Lung & 1043 & 1 & 1250.8 & 54.52 & 1305.3 & 4.2 & 0.64 & 1.08 & 1.92 \\
\hline Spleen & 900 & 1 & 1186.8 & 96.35 & 1283.2 & 7.5 & 0.53 & 1.31 & 2.12 \\
\hline Tumor & 167 & 1 & 810.4 & 102.44 & 912.8 & 11.2 & 0.15 & 4.73 & 7.43 \\
\hline
\end{tabular}

${ }^{a} * \mathrm{ng} \mathrm{ml}{ }^{-1} ;{ }^{\#}\left(\mathrm{ng} \mathrm{h} \mathrm{ml}{ }^{-1}\right) ; C_{\max }=$ maximum concentration; $T_{\max }=$ time of the peak concentration; $\mathrm{AUC}_{(0-\mathrm{t})}, \mathrm{AUC}_{0-\infty}, \mathrm{AUC}_{\mathrm{extra}}=$ areas under concentration-time curve; $T_{1 / 2}=$ terminal elimination half-life; MRT $=$ mean residence time; $L_{\mathrm{z}}=$ elimination rate constant.

intravenous route to nude mice strain bearing an orthotopic mammary tumor (MDA-MB-231). Animals administered with Block-MNP-FA (not containing PTX) were employed as control group. Block-P-MNP-FA exhibited a clear anti-tumoral activity,

A)

\section{TUMOR GROWTH}

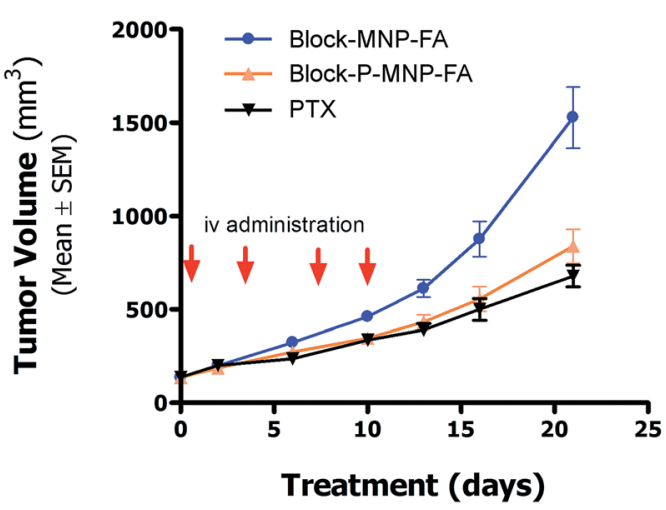

B)

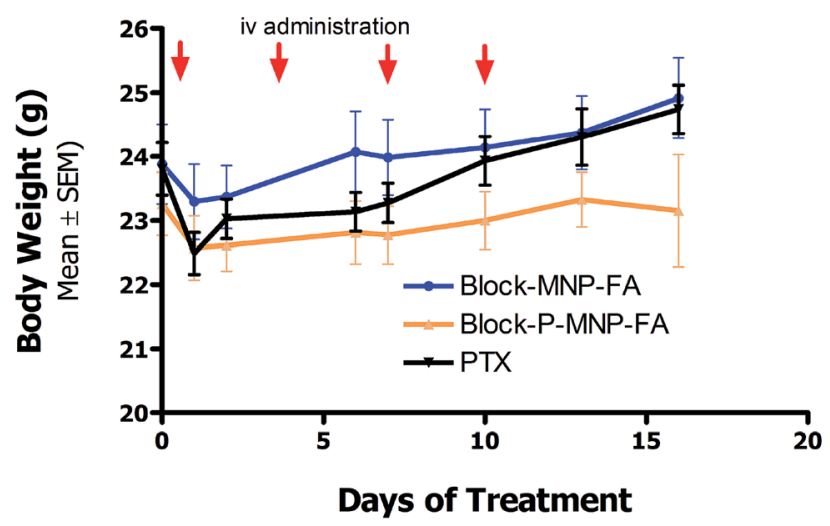

Fig. 4 Tumor volume growth (a) and body weight monitoring (b) after i.v. administration of targeted hybrid NPs (Block-MNP-FA and BlockP-MNP-FA) and commercial PTX. comparable to that of commercial PTX, with a progressive significant reduction on tumor growth rate (Fig. 4).

The i.v. administration (day 0, 3, 7 and 10) of PTX and BlockP-MNP-FA induced only a slight body weight reduction after the first dose. In case of Block-P-MNP-FA the body weight was not recovered at the end of the study (16 days), corresponding to 6 days after the last injection (day 10). The carrier not containing the PTX but loaded only with MNP displayed no effect in reducing the tumor growth and a negligible effect on mice body weight; thus confirming the tolerability of the NPs. The results of the in vivo tumor inhibition confirmed that the PTX loaded in the NPs was efficiently released and explicated its effect with an antitumoral efficacy comparable to that of commercial PTX, despite the co-presence with MNPs.

\section{In vivo MR imaging and histology}

Our MRI experiments were performed with a number of animals typical of proof-of-concept studies. In this type of experiments, the functional MNPs can be considered as efficient targeting agents, if the images at early time point and $1 \mathrm{~h}$ after the injection are very similar to the ones at $24 \mathrm{~h}$, and all images (generally $T_{2}^{*}$-weighted to highlight the so-called susceptibility effect) show dark zones inside tumors, demonstrating that the MNPs have reached the targeted tissue. ${ }^{31,33}$ Subsequently 10 female homozygote nude mice carrying subcutaneous MDA-MB-231 tumor underwent MRI at $4.7 \mathrm{~T}$ before and after the injection of the NPs ( $1 \mathrm{~h}$ and $24 \mathrm{~h}$ ). For the histological studies, the animals were sacrificed at $24 \mathrm{~h}$ and 11 days after injection.

Relevant differences were observed in FR- $\alpha$ MDA-MB-231 tumor bearing mice treated with Endorem ${ }^{\circledR}$ and Block-PMNP-FA (Fig. 5), or Endorem ${ }^{\circledR}$ and Block-MNP, the MNPs without FA functionalization (Fig. 6). In the MR $T_{2}^{*}$-weighted images of a mouse treated with Endorem ${ }^{\circledR}$ a slight loss of signal is appreciable after $1 \mathrm{~h}$ of injection (see Fig. 5, upper line), while a marked loss of signal is detectable in the case of Block-P-MNPFA treated mice (Fig. 5, lower line). However, at this time point the difference between the enhancement of the signal intensity (SI) of Endorem and Block-P-MNP-FA groups was not statistically significant. 

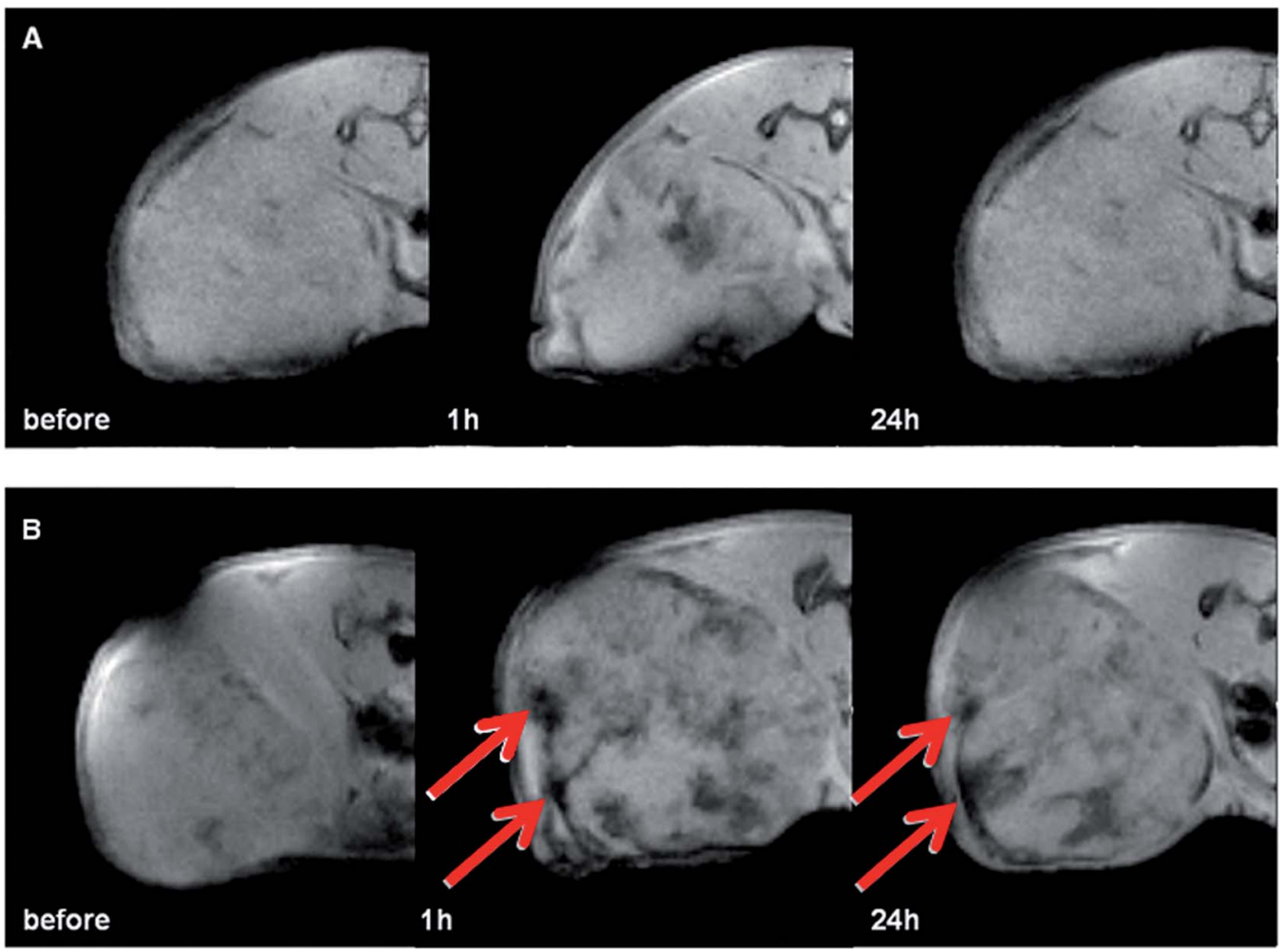

Fig. $5 \mathrm{MRI}$ of MDA-MB-231 bearing mice treated with Endorem ${ }^{\circledR}$ (Upper line) and Block-P-MNP-FA (Lower line). The arrows mark the regions reached by the NPS.
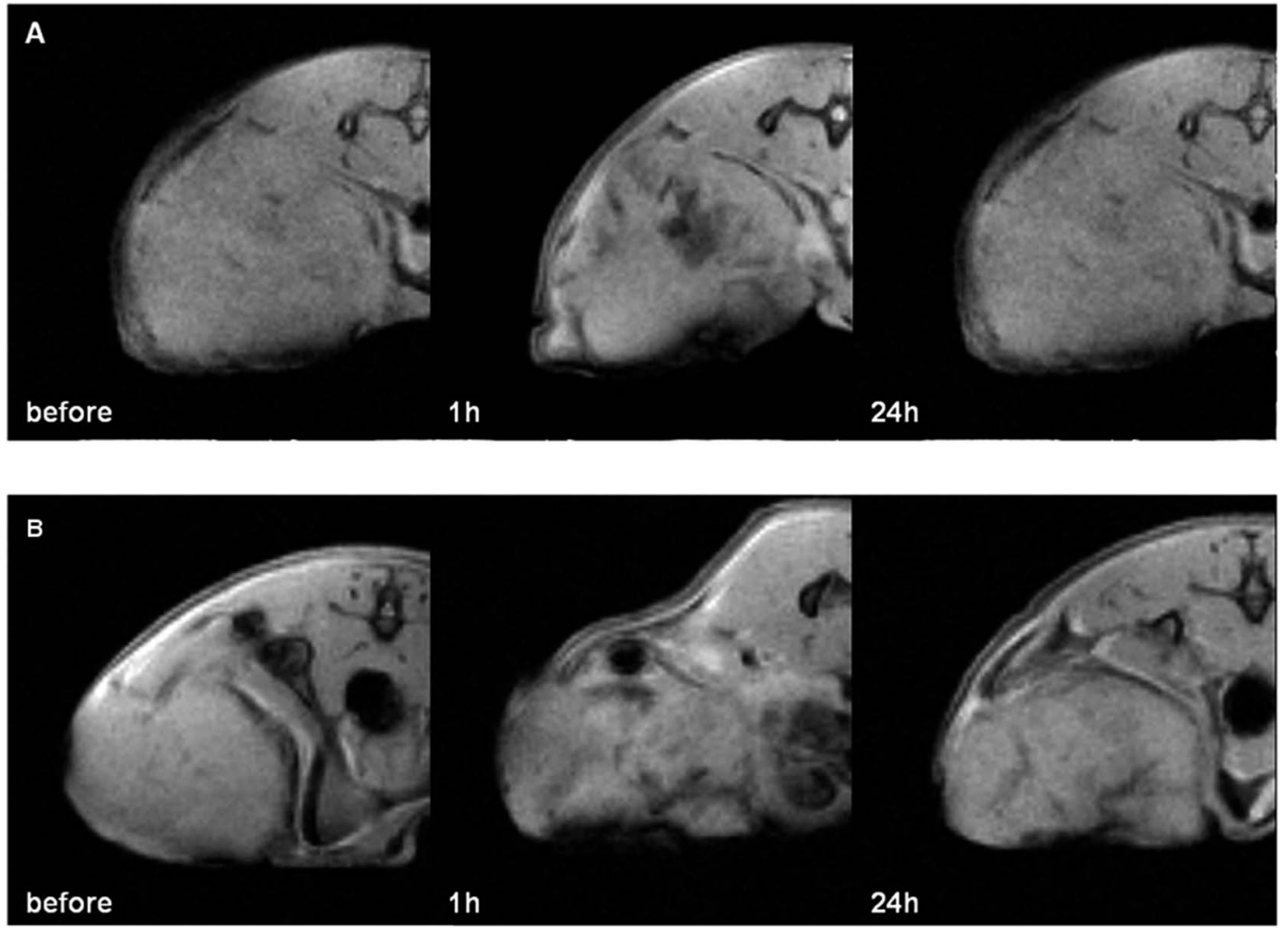

Fig. 6 MRI of MDA-MB-231 bearing mice treated with Endorem ${ }^{\circledR}$ (Upper line) and Block-P-MNP (Lower line). 
Additionally, it was observed a strong loss of signal in the tumor also $24 \mathrm{~h}$ after the injection, only for the Block-P-MNP-FA treated group, while the signal of the tumor images acquired 24 $h$ after the injection for the Endorem ${ }^{\circledR}$ treated group recovers toward the pre-injection level of intensity. At this time point, the enhancement of the SI in Block-P-MNP-FA group (-36 $\pm 3.5 \%$, mean \pm SEM) was statistically different from the value in Endorem ${ }^{\circledR}$ group $(-12 \pm 6.1 \%, p<0.05)$. This evidence indicates the higher specificity of FA present on iron oxide nanoparticles in MDA-MB-231 identification.

In the tumor masses of mice treated with the MNPs not functionalized with FA the loss of signal remained mainly restricted to the peritumoral area, indicating an accumulation in blood vessels that surround tumors and presenting a behavior not substantially different from Endorem ${ }^{\circledR}$ (Fig. 6).

In the animals monitored for prolonged time up to eleven days after the injection, the effect of PTX on tumor growth rate was detectable. The tumor volume increased by about $120 \%$ in animals administered with Endorem ${ }^{\circledR}$ and by $70 \%$ in animals administered with Block-P-MNP-FA. These results confirm the therapeutic activity of the PTX-functionalized NPs against the tumor; it can be additionally observed that the low sensitivity of the MDA-MB-231 model to PTX treatments is balanced by an uptake effect, thanks to folate targeting. ${ }^{28}$

Therefore in vivo MRI experiments confirmed that the developed NPs was acting as negative CA and was capable to target the tumor masses in MDA-MB-321 tumor bearing mice. Indeed, the specificity of folate targeting to FR- $\alpha(+)$ made the MDA-MB-231 model sensitive to the Block-P-MNP-FA system. Further studies have to evaluate the variability of tumor enhancement in a larger number of subjects as well as in cancers of different grades and sizes.

Histological analysis performed on MDA-MB-321 tumors showed an efficient delivery in the tumor mass, where is possible to distinguish two different types of areas: the first characterized by living cells markedly stained with eosin, and

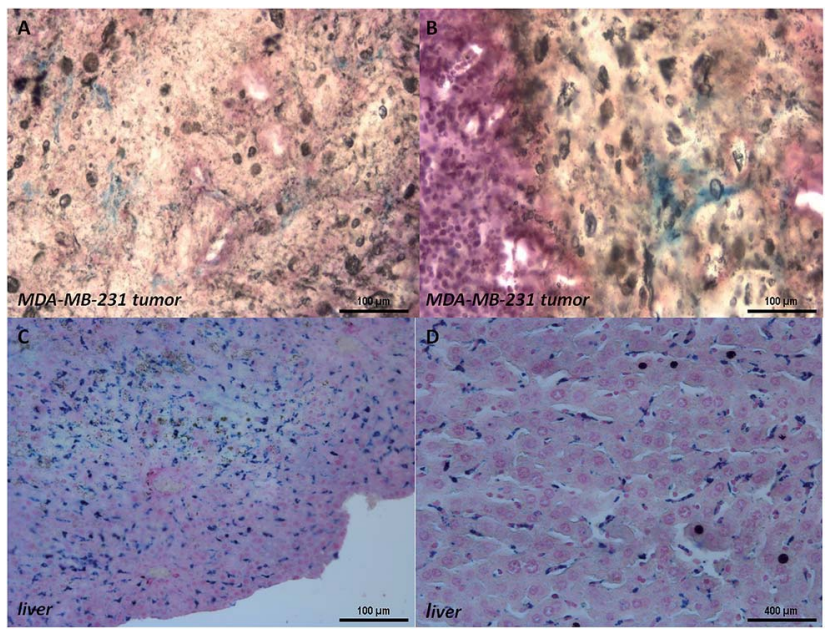

Fig. 7 Histology of MDA-MB-231 tumors and livers. In the panels (A) and (B) slices of tumors treated with NPs functionalized with FA and PTX are reported. Panels (C) and (D) showed liver sections. the second characterized by death cells not stained with eosin. The necrotic areas due to the effect of PTX are distributed in different portions of the tumors (Fig. 7A and B). In fact the regions characterized by cell apoptosis are well visible due to the lower uptake of eosin, in comparison with the living region visible in panel B on the left. The effect of PTX in tumor tissue is probably amplified by the specific targeting mediated by FA present on NPs. Iron oxide depots are also visible due to the Prussian Blue staining. The retention of iron particles in the liver is also appreciable (Fig. 7C and D), but no damaged tissue is revealed.

The specific uptake was confirmed by the in vivo MRI experiments and biodistribution studies (data not reported), as well as the content of PTX in tumor tissue indicated that the folate decorated NPs were effective in allowing the PTX uptake in tumor tissues and also in prolonging the mean residence time and the half-life elimination from the targeted tissue.

\section{Conclusions}

The proposed NPs decorated with folate show targeting ability (demonstrated by a clear uptake visible in MRI images) and a prolonged retention in FR- $\alpha(+)$ MDA-MB-231 tumor tissues. Considering the presence of PTX, the nanocarrier may be proposed also for therapeutic purposes. Our results highlight the potential in improving the PTX based cancer therapy and reducing the PTX-associated systemic toxicity, with the complementary visualization of the tumor masses through MRI technology. In mice administered with the new synthesized NPs, the increase of the tumor volume resulted nearly $40 \%$ lower compared with mice administered with Endorem ${ }^{\circledR}$, demonstrating the therapeutic efficacy of the nanocarrier. In addition, the slow and prolonged clearance of PTX from the tumor tissues after administration of Block-P-MNP-FA nanoparticles could be further exploited in local therapy of breast cancer, where the tumor cells would be exposed to PTX for longer times and greater antitumor activity would be feasible. In this view, the possibility of augment the antitumoral effect by MNP thermo-treatments will be object of future investigations.

\section{Materials and methods}

\section{Reagents}

All reagents and solvents were purchased from Sigma Aldrich unless otherwise specified. Dimethyl sulfoxide (DMSO) was refluxed over calcium hydride and distilled at reduced pressure $\left(24^{\circ} \mathrm{C} / 0.2 \mathrm{mbar}\right)$. Paclitaxel (PTX) was purchased from Discovery Fine Chemicals. Poly(ethylene glycol)-co-poly( $\varepsilon$-caprolactone) copolymer (PEGCL: Mn 35 757g mol $\mathrm{gl}^{-1}$, PI 1.9, PCL/PEG: 2.9) was provided by ARGUS Chemicals srl, the folate terminated copolymer (PEGCL-FA: Mn $15240 \mathrm{~g} \mathrm{~mol}^{-1}$, PI 1.8, PCL/PEG: 2.5, $10 \%$ wt of folate) was synthesized as previously reported (see Scheme 1) in Piras et al. $2014 .^{28}$ Briefly, PCLdiol (Mn by GPC 1500-2500, softening point $50{ }^{\circ} \mathrm{C}$, Sigma-Aldrich) and PEGdiol (average Mn 1963, melting point 53.9-55.1 ${ }^{\circ} \mathrm{C}$, Sigma-Aldrich) were dried by azeotropic distillation with toluene and further dried under vacuum before use. The terminal folate-blocks were 
synthesized in DMSO at $25{ }^{\circ} \mathrm{C}$ by coupling FA and 2 (2-amino ethoxy)ethanol in presence of $N, N^{\prime}$-dicyclohexylcarbodiimide (DCC). For the synthesis of Folate-functionalised PEGCL poly (ether ester urethane)s, PEG and PCL diols in ratio 1/3 w/w were thoroughly dried and mixed under vacuum at $60{ }^{\circ} \mathrm{C}$ for $24 \mathrm{~h}$. A stoichiometric amount of HMDI was added to the melted mixture and stirred for 3 hours at $70{ }^{\circ} \mathrm{C}$ under anhydrous conditions. Distilled DMSO was then added to dissolve the formed copolymer and continue the reaction with an excess the previously recovered folate-blocks, in presence of stoichiometric amounts of Triethylamine (TEA). The reaction was continued for $1 \mathrm{~h}$ at $70{ }^{\circ} \mathrm{C}$ under anhydrous conditions and ended by cooling under nitrogen flow. The functionalised copolymer was purified by dialysis (RC membrane MWCO 3500) against phosphate buffer $1 \mathrm{mM} \mathrm{pH} 7.8$ and subsequently against water, and recovered by freeze-drying. The product was isolated by double precipitation from chloroform into cold petroleum ether and in diethyl ether, followed by one precipitation from acetone into methanol/diethyl ether $1: 4 \mathrm{v} / \mathrm{v}$, and dried until constant weight.

Magnetite nanoparticles (average diameter $12 \mathrm{~nm}$ ) $0.93 \% \mathrm{wt}$ suspended in diethylene glycol were provided by Colorobbia Italia S.p.A. Cell line balb/3T3 Clone A31 mouse embryo fibroblast (CCL163) was purchased from American Type Culture Collection (ATCC) and propagated as indicated by the supplier; Dulbecco's Modified Eagles Medium (DMEM), 0.01 M pH 7.4 phosphate buffer saline without $\mathrm{Ca}^{2+}$ and $\mathrm{Mg}^{2+}$ (PBS), calf serum (CS), glutamine and antibiotics (penicillin/streptomycin)

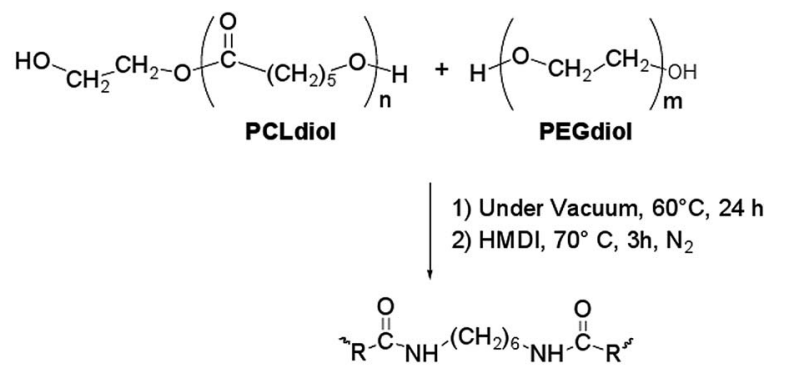

PEGCL poly(ether ester urethane)s $\mathrm{R}=\mathrm{PCLdiol}$ or PEGdiol

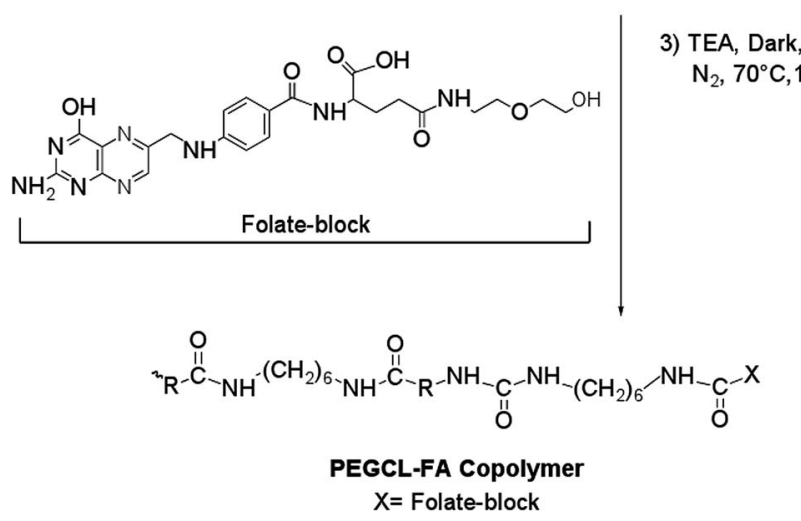

Scheme 1 Synthetic scheme for the obtainment of PEGCL-FA copolymer. were purchased from GIBCO-BRL; cell proliferation reagent WST-1 was provided by Roche Diagnostic.

The human breast adenocarcinoma cell line MDA-MB-231 was purchased from ATCC and propagated following the instructions provided by the supplier. Complete Minimum Essential Medium (MEM) (Invitrogen) was prepared by adding penicillin (100 $\left.\mathrm{U} \mathrm{ml}^{-1}\right)$, streptomycin $\left(100 \mu \mathrm{g} \mathrm{ml}^{-1}\right)$, glutamine ( $2 \mathrm{mM}$ ), sodium pyruvate $1 \mathrm{mM}$ and $10 \%$ fetal bovine serum (FBS) (Sigma) to MEM. 4'-6-diamidino-2-phenylindole (DAPI) was purchased from Invitrogen. Paraformaldehyde was purchased from Carlo Erba (Milano, Italy).

\section{Physical and morphological characterization of nanoparticles}

Dimensional analyses were carried out by Coulter LS230 Laser Diffraction Particle Size Analyzer (Beckman Coulter Fullerton, CA, USA), equipped with small volume module plus and using the Polarization Intensity Differential Scattering (PIDS) system. Diameter distribution was processed using Mie optical model and three runs were performed on each sample. NPs morphology was analysed by means of Atomic Force Microscopy (AFM). The Microscope was equipped with a type-E scanner stage allowing for measures up to $13 \times 13 \mu \mathrm{m}$ in the $X Y$ plane and about $3.5 \mu \mathrm{m}$ in the $Z$ axis. AFM images were collected using both RTESP and Olympus Biolever probes. Tapping - mode AFM was used to study the structure of NPs deposited onto freshly cleaved HOPG substrate. Zeta potential analyses were carried out by using a Delsa ${ }^{\mathrm{TM}}$ NanoC (Beckman Coulter Fullerton, CA, USA), all samples were analysed in triplicate at $25{ }^{\circ} \mathrm{C}$, using $3 \mathrm{mM} \mathrm{NaCl} \mathrm{pH} 7.4$ water solutions as dispersant. Drug loading efficacy (LE) and encapsulation efficiency (EE) were evaluated on purified NPs re-dispersed in water and lyophilized. PTX loading was evaluated by means of high performance liquid chromatography (HPLC, Perkin Elmer Series 200 - Total Chrom Navigator) by using C-18 Discovery $(25 \mathrm{~cm} \times 4.6 \mathrm{~mm} \times 5 \mathrm{~mm})$ column, isocratic elution with water and acetonitrile (61/39), UV

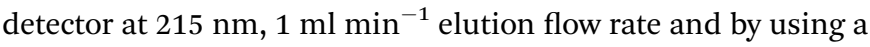
standard curve [250-7.8 $\left.\mu \mathrm{g} \mathrm{ml}^{-1}, R^{2}=0.9998\right]$ as reference. LE was defined as weight percentage of drug in purified, dried NPs. EE was defined as percentage of recovered drug in purified dried NPs with respect to the total amount of drug employed in the formulation. The amount of FA grafted to the copolymer was evaluated by means of UV-Vis absorbance at $359 \mathrm{~nm}$ (Standard concentrations 2-30 $\left.\mu \mathrm{g} \mathrm{ml} \mathrm{m}^{-1}, R^{2}=0.9997\right)$. Magnetite loading was evaluated by ICP and expressed as percentage of NPs dry weight. Student $t$-test was applied as $p<0.05$ or $p<0.01$, depending on the analysed property.

\section{Relaxometric characterization}

The ${ }^{1} \mathrm{H}$ NMR relaxometry characterization, namely NMRdispersion profile, was performed at physiological $\left(37^{\circ} \mathrm{C}\right)$ and room temperature by measuring the longitudinal and the transverse nuclear relaxation times $T_{1}$ and $T_{2}$, in the frequency range $10 \mathrm{kHz}-60 \mathrm{MHz}$. The NMR signal detection and generation was obtained with a Smartracer® Fast-Field-Cycling relaxometer (Stelar, Mede, Italy) for $10 \mathrm{kHz}-10 \mathrm{MHz}$, and with a Stelar Spinmaster spectrometer for $10-60 \mathrm{MHz}$. The nuclear 
longitudinal relaxation time $T_{1}$ was measured by means of a saturation recovery sequence $90^{\circ}-90^{\circ}$, with delay times according to the range investigated and $90^{\circ}$-pulse of about $2-5$ microsec. The nuclear transverse relaxation time $T_{2}$ was measured by a conventional Carr-Purcell-Meiboom-Gill (CPMG) sequence. Relaxation data were analyzed by means of the Stelar Company (Mede, Italy) software or homemade software, and Origin (Microcal). To determine the efficiency of the MNPs as MRI contrast agents in vitro, the longitudinal $\left(r_{1}\right)$ and transverse $\left(r_{2}\right)$ nuclear relaxivities were calculated and the obtained values at the most used clinical fields i.e. $\sim 0.2 \mathrm{~T}(9.5 \mathrm{MHz}), 0.5 \mathrm{~T}(\sim 21$ $\mathrm{MHz})$ and $\sim 1.5 \mathrm{~T}(60 \mathrm{MHz})$ were reported in the text.

\section{In vitro assessment of cytotoxicity}

Cytotoxicity evaluation of NPs was carried out using the balb/ 3T3 Clone A31 cell line. Cells were grown in DMEM containing $10 \%(\mathrm{v} / \mathrm{v})$ Calf Serum, $4 \mathrm{mM}$ glutamine, $100 \mathrm{U} \mathrm{ml}^{-1}$ of penicillin and $100 \mu \mathrm{g} \mathrm{m} \mathrm{m}^{-1}$ of streptomycin. A subconfluent monolayer of balb/3T3 cells was trypsinized using a $0.25 \%$ trypsin-1 mM EDTA solution, centrifuged at $200 \mathrm{~g}$ for $5 \mathrm{~min}$, resuspended in growth medium and counted. Appropriate dilution was made in order to obtain $2.5 \times 10^{3}$ cells per $100 \mu \mathrm{l}$ of medium, the final volume present in each well of a 96 well plate. Cells were incubated at $37{ }^{\circ} \mathrm{C}, 5 \% \mathrm{CO}_{2}$ for $24 \mathrm{~h}$ until $60-70 \%$ confluence was reached. The medium was removed from each well and replaced with complete DMEM containing purified NPs (Block, Block-MNP, or Block-MNP-FA) at two different concentrations $\left(0.1\right.$ or $0.5 \mathrm{mg} \mathrm{ml}^{-1}$ ). After $24 \mathrm{~h}$ of incubation, cells were analyzed for viability with cell proliferation reagent WST-1. Cells were incubated with WST-1 reagent diluted $1: 10$ (as indicated by the manufacturer) for $4 \mathrm{~h}$ at $37{ }^{\circ} \mathrm{C}, 5 \% \mathrm{CO}_{2}$. Plates were then analyzed with a Bio-Rad Benchmark Microplate Reader (Bio-Rad, Hercules, CA, USA); measurements of formazan dye absorbance were carried out at $450 \mathrm{~nm}$, with the reference wavelength at $620 \mathrm{~nm}$.

\section{In vivo biodistribution in tumor bearing mice}

6 groups of MDA-MB-231 tumor bearing mice (20 animals) were treated with Block-P-MNP-FA. $10 \mathrm{mg} \mathrm{ml}^{-1} \mathrm{NP}$ suspension (corresponding to $0.25 \mathrm{mg} \mathrm{ml}^{-1}$ of PTX) was injected into female mice through the tail vein, at dose of $1 \mathrm{mg}$ PTX/kg (app. $0.1 \mathrm{ml}$ ). Animals were killed after $0.5,1,3,6,10,16$ and $24 \mathrm{~h}$ ( 3 mice per time interval, and 2 mice for the last interval). The experiment was performed in fasting conditions to eliminate the presence of external FA from food. At all time points, blood samples were collected from the jugular vein into microtubes with $5 \%$ EDTA.Na $\left(20 \mu \mathrm{L} \mathrm{ml}^{-1}\right.$ blood) and centrifuged at $4000 \mathrm{rpm}$ $\min ^{-1}, 4{ }^{\circ} \mathrm{C}$ for $10 \mathrm{~min}$ to obtain plasma samples (stored at $-20{ }^{\circ} \mathrm{C}$ ). At the same time points, the tumor tissue and vital organs (lung, heart, liver, kidney, spleen and brain) were removed. The organs, except brain and tumor were cleaned in cold saline, weighted and stored at $-20{ }^{\circ} \mathrm{C}$. The organs and tumor were homogenized at $1400 \mathrm{rpm} \min ^{-1}$ for $5 \mathrm{~min}$ using Ultra-Turax T25 homogenizer in 4 volumes of $0.07 \mathrm{M}$ phosphoric acid, and then stored at $-20{ }^{\circ} \mathrm{C}$. PTX was extracted from tissue homogenates and Plasma with $1 \mathrm{ml}$ methanol every
$0.2 \mathrm{ml}$ of sample, then the samples were centrifuged at 12000 $\mathrm{rpm}, 7^{\circ} \mathrm{C}$ for $20 \mathrm{~min}$ and the supernatant was assayed by reverse phase chromatography with tandem mass spectrometry (LCMS/MS) in MRM positive ion mode. Reference standard of PTX (Mylan) was used for the quantification of the extracts (LLOQ $=1 \mathrm{ng} \mathrm{ml} \mathrm{ml}^{-1}$ ). These results were re-calculated to $\mathrm{ng}$ equivalent on gram tissue, which expressed an affinity of PTX for the organ/tumor. The pharmacokinetic parameters were calculated by the licensed software Kinetica ${ }^{\mathrm{TM}}$ TermoFisher Scientific. The concentration profiles were fitted to a noncompartment model. Selected pharmacokinetic parameters: the maximum concentration $\left(C_{\max }\right)$, time of the peak concentration $\left(T_{\max }\right)$, areas under concentration-time curve $\operatorname{AUC}\left({ }_{0-\mathrm{t}}\right)$, $\mathrm{AUC}_{0-\infty}$, AUC extra, the terminal half-life elimination $\left(T_{1 / 2}\right)$, mean residence time (MRT) and the elimination rate constant $\left(L_{\mathrm{z}}\right)$ were evaluated.

\section{In vivo study of tumor growth inhibition}

The efficacy in tumor growth inhibition of Block-P-MNP-FA NPs was compared to that of PTX alone when administered by intravenous route to nude mice strain bearing the orthotopic mammary tumor MDA-MB-231 (45 animals). Animals administered with Block-MNP-FA NPs were employed as control group. The dose assayed was $1 \mathrm{mg} \mathrm{kg}^{-1}$ for Block-MNP-FA and $3.75 \mathrm{mg} \mathrm{kg}{ }^{-1}$ of PTX for the drug containing control (Merck Millipore, Billerica, USA) and assayed NPs formulation, in fourth i.v. administration days (days 0, 3, 7 and 10). Tumor growth and body weight evolution of each animal were measured twice a week. During all the study, the animals were observed for any change on behavior and on its general appearance besides to the body weight gain.

\section{In vivo MRI diagnosis}

The experimental protocol for in vivo experiments was chosen according to Meier et al., 2010 (ref. 31) and involved 10 female homozygote nude mice bearing subcutaneous MDA-MB-231 tumor.

MDA-MB-231 bearing mice were divided into three groups: the first one of 4 animals was treated with Endorem ${ }^{\circledR}$, the second one of 3 animals was treated with Block-P-MNP-FA and the third group of 3 animals was treated with Block-P-MNP. The MNP suspensions, at the dosage of $0.5 \mathrm{mM} \mathrm{kg} \mathrm{kg}^{-1}$, and Endorem ${ }^{\circledR}$, at dose of $0.5 \mathrm{mM} \mathrm{kg}^{-1}$ (iron content), were injected into female mice through the tail vein. Because the low iron concentration in MNP suspensions, compared with Endorem ${ }^{\circledR}$, the administration of CAs was performed by slow infusion, over

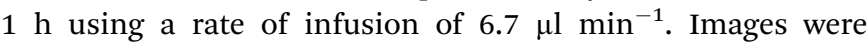
acquired before, $1 \mathrm{~h}$, and $24 \mathrm{~h}$ after the injection of the suspensions. MRI experiments were carried out using a Bruker Biospec spectrometer operating at 4.7 $\mathrm{T}$ and equipped with an actively shielded gradient system (BGA9, Bruker Germany) having a maximum gradient strength of $40 \mathrm{G} \mathrm{cm}^{-1}$. Animals were anesthetized by inhalation of a mixture of air and oxygen containing $\mathbf{0 . 5 - 1 \%}$ of Isofluorane, and placed in supine position in a $35 \mathrm{~mm}$ inner-diameter birdcage coil. A sensor for breath monitoring was positioned at the level of the chest. 
$T_{2}^{*}$-weighted sequences were acquired with the following parameters: $\mathrm{TE}=4.4 \mathrm{~ms}$, no. slices $=12$, slice thickness $=2$ $\mathrm{mm}, \mathrm{NEX}=2, \mathrm{TR}=1 \mathrm{~s}, \mathrm{FOV}=5 \times 2.5$, matrix $=256 \times 128$, resolution in space $=0.0195 \times 0.0195$. Images were analyzed by selecting three central slices in MR images of the tumor and by manually drawing in each slice two ROIs (Region-of-Interest) to cover the tumor area and to measure the SI of the skeletal muscle, respectively. The SI of the tumor was normalized to the SI of the muscle and then used to calculate the negative Enhancement of the SI according to: $E \%=100 \times($ SIpost SIpre)/SIpre, where pre and post mean respectively prior to and after administration of the contrast agent. Some animals were also monitored at longer time intervals, up to eleven days, after the injection ( $n=2$ with Endorem ${ }^{\circledR} ; n=1$ for Block-P-MNP-FA). This last group of tumor bearing mice was monitored to verify the efficacy of PTX in tumor growth slowdown. After the last MRI acquisition, animals were sacrificed for histology: tumors and livers were surgically excised and paraffin embedded. Sections of $5 \mu \mathrm{m}$ were obtained using a microtome and then stained with Prussian Blue to identify the iron NPs and successively were stained with hematoxylin and eosin to visualize nuclei and cytoplasm of the cells.

\section{Ethical animal procedures}

In vivo biodistribution. All procedures are in compliance with the Animal Welfare Act, The Guide for the Care and Use of Laboratory Animals, and the Office of Laboratory Animal Welfare. The study was approved by Ethic Committee and State Veterinary and Food Administration of the Slovak Republic Ro 2056/12-221 according to Government order 23/2009 laying down requirements for the protection of animals used for experimental purposes or other scientific purposes.

In vivo study of tumor growth inhibition. All procedures involving experimental animals were approved by the 'Ethical Committee of Animal Experimentation' of the animal facility place at Science Park of Barcelona (Platform of Applied Research in Animal Laboratory) and by the Catalonian and Spanish regulatory laws and guidelines governing experimental animal care.

In vivo MRI. The experiments received authorization from the Italian Ministry of Health and were conducted following the Italian law and the European Community Council (86/609/EEC) directive.

\section{Acknowledgements}

Authors would like to thank Dr Michele Alderighi (Department of Chemistry and Industrial Chemistry, University of Pisa) for AFM imaging. Authors would like to thank the financial support by NANOTHER project (Seventh Framework Programme: Theme NMP-2007, Large Scale Integrating Collaborative Project; no.: CP-IP 213631-2) and by INSTM-Consorzio Interuniversitario per la Scienza e Tecnologia dei Materiali through the national project FIRB "Riname".

\section{References}

1 L. Dong-Eun, K. Heebeom, S. In-Cheol, R. Ju Hee, K. Kwangmeyung and K. Ick Chan, Chem. Soc. Rev., 2012, 41, 2656-2672.

2 K. Eunjung, L. Kwangyeol, H. Yong-Min and H. Seungjoo, J. Mater. Chem. B, 2013, 1, 729-739.

3 H. Hricak, Radiology, 2011, 259, 633-640.

4 M. F. Kircher and J. K. Willmann, Radiology, 2012, 264, 349368.

5 G. Y. Lee, W. P. Qian, L. Wang, Y. A. Wang, C. A. Staley, M. Satpathy, S. Nie, H. Mao and L. Yang, ACS Nano, 2013, 7, 2078-2089.

6 L. Vannucci, E. Falvo, C. M. Failla, M. Carbo, M. Fornara, R. Canese, S. Cecchetti, L. Rajsiglova, D. Stakheev, J. Krizan, A. Boffi, G. Carpinelli, V. Morea and P. Ceci, J. Biomed. Nanotechnol., 2015, 11, 81-92.

7 S. Q. Liu, N. Wiradharma, S. J. Gao, Y. W. Tong and Y. Y. Yang, Biomaterials, 2007, 28, 1423-1433.

8 Y. Lu and P. S. Low, J. Controlled Release, 2003, 91, 17-29.

9 Y. Zhang, N. Kohler and M. Zhang, Biomaterials, 2003, 23, 1553-1561.

10 S. Wang, J. Luo, D. A. Lantrip, et al., Bioconjugate Chem., 1997, 8, 673-679.

11 E. S. Lee, K. Na and Y. H. Bae, J. Controlled Release, 2003, 91, 103-113.

12 S. Mansouri, Y. Cuie, F. Winnik, Q. Shi and P. Lavigne, Biomaterials, 2006, 27, 2060-2065.

13 M. O. Oyewumi, R. A. Yokel, M. Jay, T. Coakley and R. J. Mumper, J. Controlled Release, 2004, 95, 613-626.

14 H. Z. Zhao, L. Yue and Y. Lanry, Int. J. Pharm., 2008, 349, 256268.

15 Z. Yang, S. Kang and R. Zhou, Nanoscale, 2014, 6, 663-677. 16 Q. A. Pankhurst, N. T. K. Thanh, S. K. Jones and J. Dobson, J. Phys. D: Appl. Phys., 2009, 42, 224001.

17 A. G. Roca, R. Costo, A. F. Rebolledo, S. VeintemillasVerdaguer, P. Tartaj, T. González-Carreño, M. P. Morales and C. J. Serna, J. Phys. D: Appl. Phys., 2009, 42, 224002.

18 B. H. Kim, M. J. Hackett, J. Park and T. Hyeon, Chem. Mater., 2014, 26, 59-71.

19 A. K. Suresh, D. A. Pelletier and M. Doktycz, Nanoscale, 2013, 5, 463-474.

20 E. Poselt, H. Kloust, U. Tromsdorf, M. Janschel, C. Hahn, C. Maßlo and H. Weller, ACS Nano, 2012, 6, 1619-1624.

21 L. Xiao, J. Li, D. F. Brougham, E. K. Fox, N. Feliu, A. Bushmelev, A. Schmidt, N. Mertens, F. Kiessling, M. Valldor, B. Fadeel and S. Mathur, ACS Nano, 2011, 5, 6315-6324.

22 N. Lee, Y. Choi, Y. Lee, M. Park, W. K. Moon, S. H. Choi and T. Hyeon, Nano Lett., 2012, 12, 3127-3131.

23 (a) P. Arosio, J. Thévenot, T. Orlando, F. Orsini, M. Corti, M. Mariani, L. Bordonali, C. Innocenti, C. Sangregorio, H. Oliveira, S. Lecommandoux, A. Lascialfari and O. Sandre, J. Mater. Chem. B, 2013, 1, 5317-5328; (b) V. Amendola, M. Meneghetti, O. M. Bakr, P. Riello, S. Polizzi, D. H. Anjum, S. Fiameni, P. Arosio, T. Orlando, 
C. d.-J. Fernandez, F. Pineider, C. Sangregorio and A. Lascialfari, Nanoscale, 2013, 5, 5611-5619.

24 (a) Y. Xu, W. E. Heberlein, M. Mahmood, A. I. Orza, A. Karmakar, T. Mustafa, A. R. Biris, D. Casciano and A. S. Biris, J. Mater. Chem., 2012, 22, 20128-20142; (b) B. Du, S. Han, H. Li, F. Zhao, X. Su, X. Cao and Z. Zhang, Nanoscale, 2015, 7, 5411-5426.

25 D.-E. Lee, H. Koo, I.-C. Sun, J. H. Ryu, K. Kim and I. C. Kwon, Chem. Soc. Rev., 2012, 41, 2656-2672.

26 L. Lartigue, C. Innocenti, T. Kalaivani, A. Awwad, M. M. Sanchez, Y. Guari, J. Larionova, C. Guérin, J. L. Montero, V. Barragan, P. Arosio, A. Lascialfari, D. Gatteschi and C. Sangregorio, J. Am. Chem. Soc., 2011, 133, 10459-10472.

27 E. Kim, K. Lee, Y.-M. Huh and S. Haam, J. Mater. Chem. B, 2013, 1, 729-739.
28 A. M. Piras, S. Sandreschi, S. Ponnurengam Malliappan, et al., Int. J. Pharm., 2014, 475, 523-535.

29 S. C. Kim, D. W. Kim, Y. H. Shim, et al., J. Controlled Release, 2001, 72, 191-202.

30 (a) A. K. Singla, A. Garg and D. Aggarwal, Int. J. Pharm., 2002, 235, 179-192; (b) D. Liu, W. Wu, X. Chen, S. Wen, X. Zhang, Q. Ding, G. Teng and N. Gu, Nanoscale, 2012, 4, 2306-2310.

31 R. Meier, T. Henning, S. Boddington, et al., Radiology, 2010, 255, 527-535.

32 C. Galindo-González, J. de Vicente, M. Ramos-Tejada, M. López-López, F. González-Caballero and J. Durán, Langmuir, 2005, 21, 4410-4419.

33 K. Tao, S. Song, J. Ding, H. Dou and K. Sun, Colloid Polym. Sci., 2011, 289, 361-369.

34 P. Marzola, A. Degrassi, L. Calderan, et al., Clin. Cancer Res., 2004, 10, 739-750. 